J. Schipper ${ }^{1} \cdot$ T. Klenzner $^{1} \cdot$ A. Berlis ${ }^{2} \cdot$ W. Maier ${ }^{1} \cdot$ C. Offergeld ${ }^{1}$.

A. Schramm ${ }^{3} \cdot$ N.-C. Gellrich ${ }^{3}$

${ }^{1}$ Universitätsklinik für Hals-, Nasen- und Ohrenheilkunde und Poliklinik,

Universitätsklinikum Freiburg

${ }^{2}$ Sektion Neuroradiologie des Neurozentrums, Universitätsklinikum Freiburg

${ }^{3}$ Klinik für Mund-, Kiefer- und Gesichtschirurgie, Medizinische Hochschule Hannover

\title{
Objektivierung von Therapieergebnissen in der Schädelbasis- chirurgie durch virtuelle Modellanalyse
}

Die Verwendung neuer Algorithmen in der medizinischen Navigation erlaubt es, in immer kürzerer Zeit in planaren Bildserien von dreidimensionalen computertomographischen (CT-) und magnetresonanztomographischen (MR-)Datensätzen Umrisse interessierender anatomischer Strukturen zu segmentieren und virtuell dreidimensional zu projizieren $[1$, 2]. Mit solchen virtuellen Modellen lassen sich anhand radiologischer Datensätze kubische Oberflächen oder Räume von Tumoren oder Defekte im Schädelbasisbereich kongruent dreidimensional skizzieren [1].

Bei der dreidimensionalen Projektion virtueller Modelle werden sämtliche CToder MR-Datensätze in einem Bild visualisiert. Damit ergibt sich im Vergleich zur planaren Darstellung in axialer, koronarer oder sagittaler Schichtung eine andere Bildinformation ( $\bullet$ Abb. 1): In nur einem Bild lässt sich der Zusammenhang zwischen Tumor-bzw. Defektgrenzen und der umgebenden Anatomie für die Therapieplanung räumlich analysieren und somit beispielsweise das Therapieergebnis anhand dieses Datensatzes navigiert objektivieren [13]. Ebenso ermöglicht die virtuelle Modellanalyse eine Volumen- berechnung, beispielsweise von Tumoren. Damit lässt sich sowohl das Tumorvolumen als auch der Verlauf im Rahmen eines Downstagings mit Hilfe der Bildfusion objektiv überwachen.

Bei der dreidimensionalen Visualisierung kommt es jedoch zu einem scheinbaren Verlust der Detailtreue nichtsegmentierter Bereiche. Hauptgrund hierfür ist nicht die Ungenauigkeit des verwendeten Algorithmus, sondern das gezielte Weichzeichnen und der Grauwertausgleich bei der Überlagerung hintereinander liegender Strukturen zugunsten des segmentierten Objektes. Sowohl die Blickperspektive des Betrachters, der Grad der Überblendung und der Grauwertausgleich lassen sich bei der Modellanalyse im Planungs- und Simulationmodus manuell einstellen.

Intraoperativ ist daher für eine detailgetreue computerassistierte Punktdarstellung sämtlicher anatomischer Strukturen immer einer planaren Visualisierung in axialer, koronarer und sagittaler Schichtung der Vorzug zu geben. Trotz des immer noch nicht zu unterschätzenden zusätzlichen Zeitaufwandes für das Segmentieren in den einzelnen planaren Datensätzen [15] ergeben sich Indikationen für die virtuelle Modellanalyse im Bereich der Schädelbasischirurgie. Ein Ziel ist hier die Objektivierung von Therapieergebnissen und -zielen über den zeitlichen Verlauf.

\section{Patienten und Methoden}

Für die Qualitätssicherungsanalyse wurden die Daten von 7 Patienten fallbezogen, qualitativ und deskriptiv ausgewertet. Bei allen Patienten erfolgte eine virtuelle Modellanalyse zur Objektivierung der Therapieergebnisse bei Tumoren im Bereich der anterioren und lateralen Schädelbasis. Die einzelnen Patientendaten einschließlich Tumorstaging, Histologie und Therapieregime sind in $\bullet$ Tab. 1 zusammenfassend dargestellt. Die Patienten ließen sich hinsichtlich des Therapieregimes unterteilen in Patienten mit neoadjuvanter Chemotherapie, navigiert kontrollierter chirurgischer Tumorresektion und Defektrekonstruktion (• Tab. 1, Fall 1 und 2), und Patienten mit ausschließlich navigiert kontrollierter chirurgischer Tumorresektion und/oder Defektrekonstruktion (• Tab. 1, Fall 3-7).

Alle Patienten erhielten zur Diagnosestellung und Therapieplanung gemäß unserem Standardprotokoll ein CT oder 


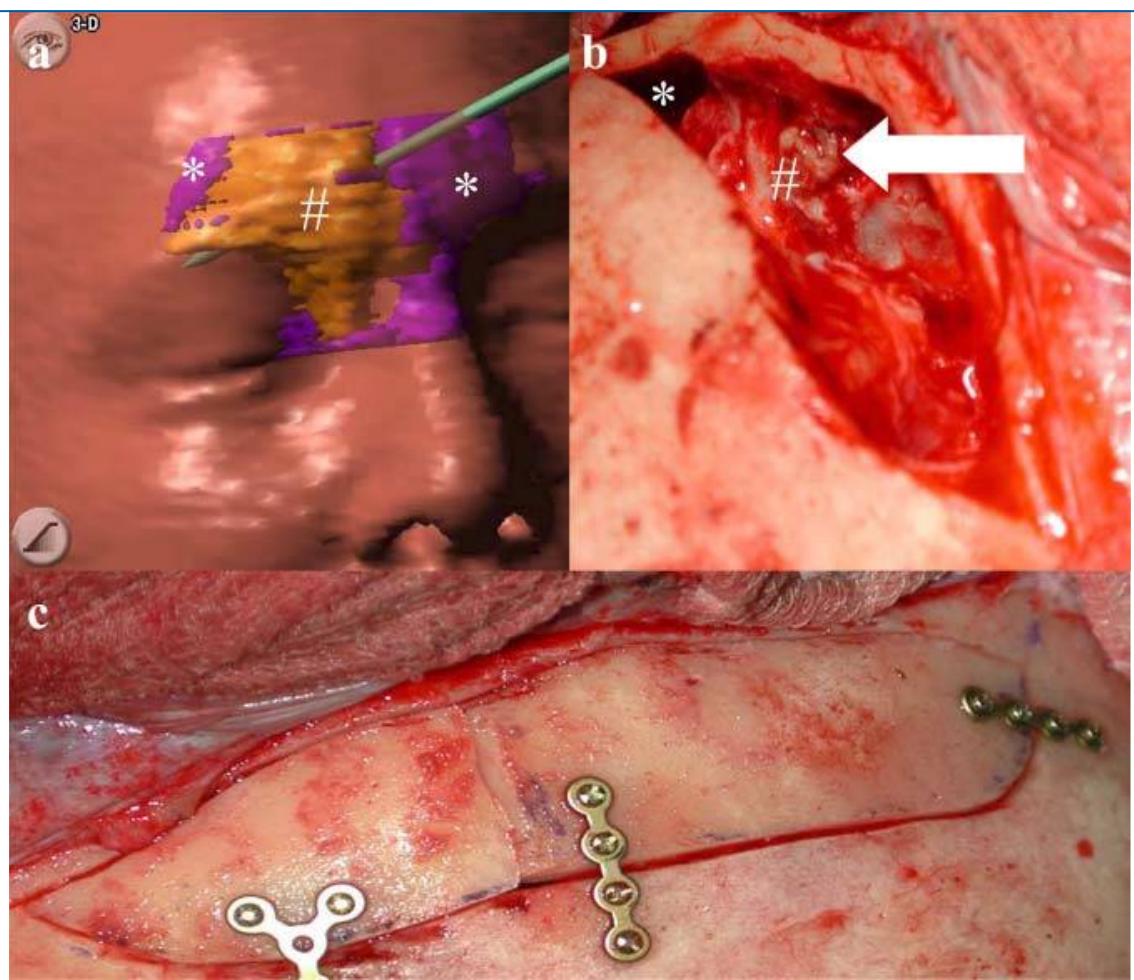

Abb. 1a-c $\Delta$ Intraoperative Visualisierung eines virtuellen Modells eines Stirnhöhlenosteoms (• Tab. 1, Fall 6) anhand eines 3D-CT-Datensatzes. a Der Pointer symbolisiert die Position des MeiBels, mit dem das Osteom von der Rückseite des zweigeteilten osteoplastischen Knochendeckels der Stirnhöhlenvorderwand abgetrennt wurde. Die optimale Position für die Zweiteilung des Knochendeckels richtete sich nach der günstigsten Winkelposition zur Lösung des Osteoms von der Rückseite der Stirnhöhlenvorderwand und wurde mit Hilfe der virtuellen Modellanalyse des Osteoms bestimmt. b Intraoperativer Situs auf das Spatium sinus frontalis (bikoronarer Hautschnitt, transfrontaler Zugang) nach Durchtrennen der knöchernen Verbindung zwischen Stirnhöhlenvorderwand und Osteom (• Tab. 1, Fall 6). Der Pfeil zeigt auf die knöcherne Basis des Verbindungsstücks zwischen Osteom und Rückseite der Stirnhöhlenvorderwand. c Intraoperativer Situs nach Wiedereinsetzen des zweigeteilten osteoplastischen Knochendeckels (• Tab. 1, Fall 6). * Spatium sinus frontalis, \# Osteom

MRT. Die Durchführung der Schichtbilduntersuchungen erfolgte nach Vorgaben der computerassistierten Chirurgie (CAS) (CT-Spiraltechnik Siemens Somatom, Schichtdicke $1 \mathrm{~mm}$, Matrix $512 \times 512$ bei einer Pixelgröße zwischen $0,41 \mathrm{~mm} 2$ und o,69 mm2 oder 1,5 T MR General Electric SIGNA System, Schichtdicke 1,5-1,7 mm, Matrix 256×256). Die Schichtbilduntersuchungen umfassten das gesamte Neuround Splanchnokranium.

Als Navigationssystem dienten das System der Fa. Stryker-Leibinger (NavigationSystem $^{\circledast}$ ) und der Fa. BrainLAB (VectorVision $2^{\circledR}$ ). Die Registrierung des Patientenkopfs erfolgte mit einer lasergestützen Oberflächenreferenzierung des Splanchnokraniums (System BrainLAB; [12]) oder mit einem individuell angefertigten markergestützen Oberkieferschienen-System (System Stryker-Leibinger; $[6,16])$. Als Tracker für den Patien- tenkopf diente ein am Patientenschädel fixierter Dreipunktstern (BrainLAB) oder ein an die Mayfield-Klemme gekoppeltes System (Stryker-Leibinger; $[4,11]$ ).

Die Segmentierung erfolgte manuell im Planungsmodus am entsprechenden Navigationssystem in sämtlichen zu analysierenden Schichten. Konturiert wurden die Umrisse interessierender Pathologien im Schädelbasisbereich wie Tumoren (• Tab. 1, Fall 1-7) oder zusätzlich Grenzflächen nativer Körperhöhlen wie beispielsweise die des Sinus frontalis (• Tab. 1, Fall 6) oder andere unmittelbar benachbarte vital oder funktionell relevante Gefäß- und Nervenstrukturen. Die Volumenberechnung der virtuellen Modelle ergab sich aus der Summation sämtlicher segmentierter Flächen unter Berücksichtigung der jeweiligen Schichtbilddicken über Extrapolation mit Hilfe eines Volumen-Näherungsalgorithmus.
Der interindividuelle Vergleich der Objektgrenzflächen der virtuellen Modelle erfolgte durch eine patientenkopfgetrackte Bildfusion. Zur Visualisierung der verschiedenen projizierten virtuellen Modelle nach der Bildfusion in nur einem Datensatz wurden diese mit unterschiedlichen Farben markiert.

\section{Ergebnisse}

Die Projektion virtueller dreidimensionaler Modelle erfolgte in Hinblick auf den nicht unerheblichen zusätzlichen Zeitaufwand im günstigsten Fall von 30 min (- Tab. 1, Fall 2) durch die schichtweise Einzelkonturierung der Tumorgrenzflächen nur in ausgewiesenen Behandlungsfällen. Das Patientenalter lag zum Operationszeitpunkt zwischen 17 und 68 Jahren (• Tab. 1).

Hinsichtlich des niedrigen Alters, der Tumorhistologie und Tumorlokalisation mit Infiltration der knöchernen Rhinobasis wurde in Zusammenarbeit mit dem Tumorzentrum Freiburg im Fall 1 und 2 (- Tab. 1) ein multimodales Therapiekonzept angestrebt: Zunächst wurde eine neoadjuvante Chemotherapie als Downstaging eingeleitet, jeweils mit 4 Zyklen unterschiedlicher Protokolle (• Tab. 1).

Im Fall 1 (• Tab. 1) konnte nach jedem Zyklus anhand der virtuellen Modellanalyse einschließlich Volumetrie eine Tumorregression nachgewiesen werden (• Abb. 2a-d).

Im Fall 2 (• Tab. 1) fand sich nach Ende des 2. Zyklus ein objektiver Nachweis einer Tumorregression, nach dem 3. Zyklus eine Stagnation und nach dem 4. Zyklus eine erneute Tumorprogression (- Abb. 3a-h). Jeweils nach dem 4. Zyklus führten wir interdisziplinär eine navigiert kontrollierte Tumorresektion in den originären Tumorgrenzen anhand des von uns zum Zeitpunkt der Erstdiagnose skizzierten virtuellen Tumormodells durch (• Abb. 2e,f).

Die Operation erfolgte jeweils über einen kombinierten transmaxillären und subfrontalen Zugang nach Raveh. Wir nahmen die Operation sowohl für die Tumorresektion als auch für die Rekonstruktion navigiert kontrolliert vor. Operiert wurde mit Hilfe der CAS in den originären Tumorgrenzen einschließlich 
eines onkologischen Sicherheitsabstandes (• Abb. 2e,f). Durch das präoperative Downstaging und das Operieren in den originären Tumorgrenzen konnte ein zusätzlicher „relativer“ onkologischer Sicherheitskorridor erzeugt werden, was sonst im Schädelbasisbereich durch die unmittelbare Nachbarschaft vitaler Gefäß- und Nervenstrukturen einschließlich der Hirnweichteile nicht möglich wäre.

Beide Patienten erhielten eine perkutane postoperative Strahlentherapie in Dreifeldertechnik.

Unsere aufgearbeiteten Datensätze wurden für das radiologische Tumor-Follow-up digital gespeichert. Durch Bildfusion ließen sich die zum Zeitpunkt der Erstdiagnose ursprünglichen Grenzflächen des virtuellen Tumormodells in den aktuellen Datensatz vom Tumor-Followup im Planungs- und Simulationsmodus wieder einblenden, um für die Beurteilung eines fraglichen Rezidivs die dafür in Frage kommende Tumormatrix zu visualisieren und von anderen Gewebsneubildungen wie Narben oder Schwellungen abzugrenzen (- Abb. 2g, h). Im Fall 2 (• Tab. 1) wurde mit Einverständnis der Patientin unter Berücksichtigung der biologischen Strahlendosis bei bekannter Klaustrophobie alternativ zum MR jeweils ein aktueller CT-Datensatz angefertigt.

Mit Hilfe der virtuellen Modellanalyse war es analog in den übrigen Fällen möglich, das sich radiologisch abbildende Tumorvolumen komplett zu erfassen und volumetrisch zu berechnen. Ebenso konnten beim Downstaging (• Tab. 1, Fall 1, 2) innerhalb eines Tumors lokal begrenzte Zonen mit einer Tumorprogression oder -regression im klinischen Verlauf objektiviert werden. Tumorregionen mit einer in der virtuellen Modellanalyse nachweislich lokal begrenzten Tumorprogression bei einer insgesamt volumetrisch zu berechnenden Tumorstagnation oder -regression (• Tab. 1, Fall 1) wurden als Non-Responder-Gewebe gewertet und intraoperativ möglichst weit im Gesunden radikalchirurgisch saniert. Die vergleichende virtuelle Modellanalyse diente nicht zur Beurteilung des Wachstums- oder möglichen Rezidivverhaltens von Malignomen, sondern auch intraoperativ zur Beurteilung raumfordernder

\section{J. Schipper · T. Klenzner · A. Berlis · W. Maier · C. Offergeld · A. Schramm · N.-C. Gellrich Objektivierung von Therapieergebnissen in der Schädelbasischirurgie durch virtuelle Modellanalyse}

Zusammenfassung

Hintergrund. Die patientenkopfgetrackte virtuelle Modellanalyse erlaubt die Objektivierung und das Monitoring von Therapieergebnissen im Schädelbasisbereich. Lange Rechnerzeiten haben bislang eine Einführung in die klinische Routine verhindert. Methoden. In einer Qualitätssicherungsanalyse wurden die Patientendaten von 7 Patienten unterschiedlicher Pathologien im Schädelbasisbereich nach virtueller Modellanalyse ausgewertet.

Ergebnisse. Die Bearbeitungszeit für das Segmentieren interessierender anatomischer Strukturen betrug in unserer Auswertung im günstigsten Fall 30 min. Die virtuelle Modellanalyse erlaubt das räumliche Visualisieren interessierender Strukturen zu ihrer Umgebung und verbessert somit das fallbezogene pathologisch-anatomische Verständnis für die Therapieplanung.

Schlussfolgerungen. Hinsichtlich der dysmorph verlaufenden Knochen-WeichteilGrenzflächen im Schädelbasisbereich erlaubt die virtuelle Modellanalyse derartig lokalisierter Pathologien ein verbessertes räumliches Verständnis und eignet sich zur Qualitätssicherung von Therapieergebnissen.

\section{Schlüsselwörter}

CAS · Manuelle Segmentierung · Radiologisches Tumormonitoring $\cdot$ Intraoperative Qualitätssicherung · Modellanalyse

\section{Objectivity of therapeutic results following skull base surgery using virtual model analysis}

\begin{abstract}
Background. Virtual model analysis of patient head tracking allows for objectivity and the monitoring of therapeutic results of pathologies in the skull base region. The introduction of these models in clinical routine has been impaired by the extended time needed for the preparation of radiolog ical data.

Methods. Quality control analysis was carried out for seven cases with different pathological findings in the skull base region in patients who had undergone virtual model analysis.

Results. Preparation time of radiological data for the process of segmentation required, under optimal conditions, a minimum of $30 \mathrm{~min}$. Virtual model analysis enables spatial visualization of regions of interest and ad-
\end{abstract}

jacent anatomical structures. This improves case-specific pathoanatomical understanding as well as preoperative planning of surgical strategies.

Conclusions. Virtual model analysis improves the physician's spatial comprehension of localized pathological findings at the dysmorphic interface of bone and soft tissue across the skull base. Therefore, it seems to be an adequate tool for quality control analysis of therapeutic results after extended skull base surgery.

\section{Keywords}

CAS · Manual segmentation · Radiological tumor monitoring · Intrasurgical quality assurance $\cdot$ Virtual model analysis 


\begin{tabular}{|c|c|c|c|c|}
\hline Fall & $\begin{array}{l}\text { Patient, } \\
\text { Geschlecht }\end{array}$ & $\begin{array}{l}\text { Alter bei Op. } \\
\text { [Jahre] }\end{array}$ & Diagnose & $\begin{array}{l}\text { Indikation zur navigierten } \\
\text { virtuellen Modellanalyse }\end{array}$ \\
\hline \multirow[t]{3}{*}{1} & \multirow[t]{3}{*}{ G.H., w. } & \multirow[t]{3}{*}{37} & \multirow[t]{3}{*}{$\begin{array}{l}\text { Esthesioneuro- } \\
\text { blastom, Kadish C, } \\
\text { Hyams II }\end{array}$} & $\begin{array}{l}\text { 1) Neoadjuvante Chemotherapie } \\
\text { (4 Zyklen: EVAIA-Protokoll, Etoposid, } \\
\text { Vincristin, Adriamycin, Ifosfamid, } \\
\text { Actinomycin-D) }\end{array}$ \\
\hline & & & & $\begin{array}{l}\text { 2) Tumorresektion in den originären } \\
\text { Tumorgrenzen (subfrontaler Zugang } \\
\text { nach Raveh) }\end{array}$ \\
\hline & & & & 3) Rekonstruktion \\
\hline \multirow[t]{3}{*}{2} & \multirow[t]{3}{*}{ B.S., w. } & \multirow[t]{3}{*}{37} & \multirow[t]{3}{*}{$\begin{array}{l}\text { Anaplastisches } \\
\text { NNH-CA T4aN0M0 } \\
\text { (UICC 2002) }\end{array}$} & $\begin{array}{l}\text { 1) neoadjuvante Chemotherapie } \\
\text { (4 Zyklen: Cisplatin, Epirubicin, } \\
\text { Cyclophosphamid) }\end{array}$ \\
\hline & & & & $\begin{array}{l}\text { 2) Tumorresektion in den originären } \\
\text { Tumorgrenzen („,midfacial } \\
\text { degloving“, subfrontaler Zugang } \\
\text { nach Raveh) }\end{array}$ \\
\hline & & & & 3) Rekonstruktion \\
\hline \multirow[t]{2}{*}{3} & \multirow[t]{2}{*}{ S. A., w. } & \multirow[t]{2}{*}{42} & \multirow{2}{*}{$\begin{array}{l}\text { Esthesioneuro- } \\
\text { blastom, Kadish C, } \\
\text { Hyams I }\end{array}$} & $\begin{array}{l}\text { 1) Tumorresektion (transfrontaler } \\
\text { Zugang) }\end{array}$ \\
\hline & & & & 2) Rekonstruktion \\
\hline \multirow[t]{2}{*}{4} & \multirow[t]{2}{*}{ D.E.R., w. } & \multirow[t]{2}{*}{17} & \multirow{2}{*}{$\begin{array}{l}\text { Riesenzellgra- } \\
\text { nulom der Fossa } \\
\text { infratemporalis }\end{array}$} & $\begin{array}{l}\text { 1) Tumorresektion (infratemporaler } \\
\text { Zugang) }\end{array}$ \\
\hline & & & & 2) Rekonstruktion \\
\hline \multirow[t]{2}{*}{5} & \multirow[t]{2}{*}{ K.G., m. } & \multirow[t]{2}{*}{48} & \multirow{2}{*}{$\begin{array}{l}\text { NNH-Karzinosar- } \\
\text { kom, T4aN1M0 } \\
\text { (UICC 2002) }\end{array}$} & $\begin{array}{l}\text { 1) Tumorresektion (transmaxillärer } \\
\text { Zugang nach Dieffenbach) }\end{array}$ \\
\hline & & & & 2) Rekonstruktion \\
\hline \multirow[t]{2}{*}{6} & \multirow[t]{2}{*}{ K.R., w. } & \multirow[t]{2}{*}{68} & \multirow[t]{2}{*}{ Stirnhöhlenosteom } & $\begin{array}{l}\text { 1) Tumorresektion (osteoplastische } \\
\text { Stirnhöhlenoperation) }\end{array}$ \\
\hline & & & & 2) Rekonstruktion \\
\hline 7 & S. A., m. & 40 & $\begin{array}{l}\text { Plattenepithelkar- } \\
\text { zinom der NNH, } \\
\text { T4bNOMO (UICC } \\
\text { 2002) }\end{array}$ & $\begin{array}{l}\text { 1) Tumorresektion } \\
\text { („midfacial degloving“) }\end{array}$ \\
\hline
\end{tabular}

Strukturen und deren Abgrenzung zu nativen Körperhöhlen.

Im Fall 6 (• Tab. 1) wurde ein Osteom als Ursache einer Stirnhöhlenmukozele im Bereich des rechten Sinus frontalis abgetragen. Das Osteom war sowohl mit der Stirnhöhlenvorderwand als auch der Rückwand verknöchert. Ziel der Operation war es, durch eine präoperativ genau vorausberechnete Zweiteilung des osteoplastischen Knochendeckels das Osteom durch einen möglichst günstigen Winkel von der Rückseite des Stirnhöhlenvorderwanddeckels mit einem Meißel abzuschlagen. Nur so war die Stirnhöhlenvorderwand komplett zu erhalten und osteoplastisch wieder zu verschließen. Durch die dreidimensionale virtuelle Modellanalyse konnte die optimale Position für die Zweiteilung des Knochendeckels bestimmt werden. Auf den planaren Schich- sualisierte Interpretation zweidimensionaler radiologischer Datensätze dient der Diagnosefindung, die der dreidimensionalen Darstellung der Therapieplanung und -ausführung [7]. Nur die direkte Endoskopie ist hinsichtlich der Bildinformation radiologischer $3 \mathrm{D}$-Datensätze gleichwertig. Sie ist jedoch im Vergleich dazu invasiv und erlaubt nur eine endoluminale Visualisierung von Hohlkörpern ohne die Möglichkeit, hintereinander liegende oder von Knochen oder Weichteilgewebe verdeckte Strukturen darstellen zu können. Moderne radiologische 3D-Darstellungen ermöglichen heute eine sog. „virtuelle Endoskopie“ [5].

Die Projektion dreidimensionaler virtueller Modelle setzt eine Segmentierung der relevanten anatomischen Strukturen voraus. Segmentierung meint die Isolierung einer bestimmten individuellen anatomischen Struktur in einem $3 \mathrm{D}-\mathrm{Da}-$ tensatz anhand der Pixel-Intensität nach den Hounsfield-Einheiten. Aufgrund der Inhomogenität des Gewebes, des unterschiedlichen Kontrastmittelaufnahmeverhaltens oder gleicher Pixel-Intensität des umliegenden Gewebes lassen sich die interessierenden anatomischen Strukturen nicht immer in allen Schichten durch spezielle Algorithmen automatisch abgrenzen, sodass immer noch manuell nachkorrigiert werden muss $[9,10,17]$.

Zukünftiges Ziel wäre in Hinblick auf den hohen Zeitaufwand für die manuelle Segmentierung ein vollautomatisches Verfahren, dass durch Einfügen zusätzlicher Algorithmen diese Aspekte impliziert. Ein zusätzlicher Vorteil einer vollautomatischen Segmentierung wäre eine Reduzierung der Fehlerstreuung im Rahmen der manuellen Segmentation. Noch vor 10 Jahren konnte eine manuelle Segementierung bis zu $4 \mathrm{~h}$ dauern [13]. Neue, schnellere Algorithmen und Rechnerzeiten haben diese Prozedur deutlich zeitlich reduziert, in unserer Auswertung im günstigsten Fall bis auf 30 min. Jedoch beschränkte sich in unseren Fällen die manuelle Segmentierung nicht auf sämtliche Knochen- und Weichteilstrukturen, sondern nur auf wenige Objekte wie die des Tumors, Grenzflächen natürlicher anatomischer Hohlräume, die der A. carotis interna oder des N. trigeminus, was die Bearbeitungszeit senkt. 


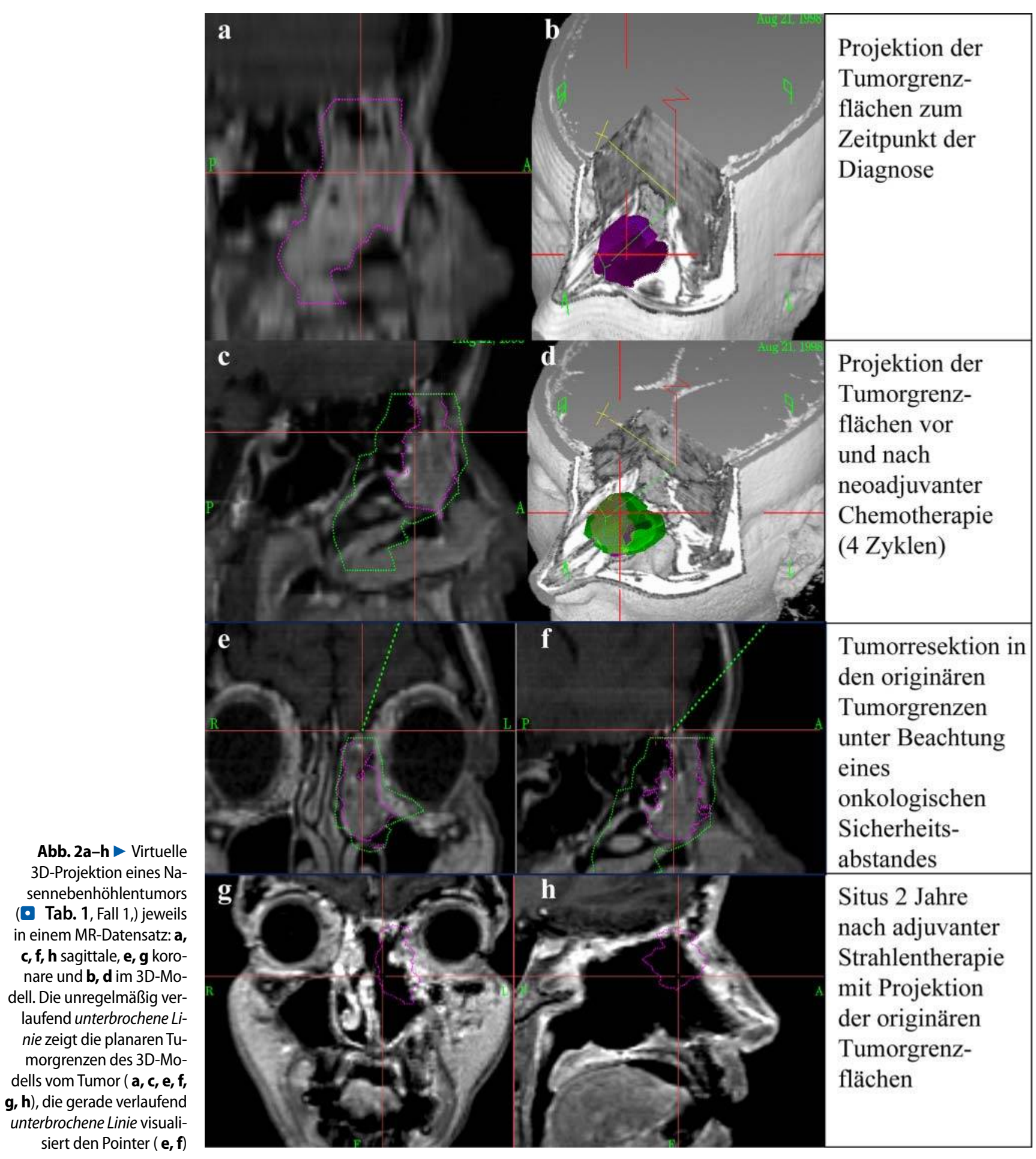

Die Autosegmentierung und dreidimensionale Darstellung der wichtigsten knöchernen Strukturen sowie der Körperoberflächen gelingt bereits in den neueren System problemlos und schnell innerhalb von 1-2 min. Allerdings beschränken sich diese Algorithmen auf die Projektion knöcherner Strukturen ohne Weichteildarstellungen.
Unsere Wahrnehmung begrenzt die Verarbeitung der in einer $3 \mathrm{D}$-Darstellung enthaltenen Informationen. So müssen für unser Verständnis von Raumwahrnehmung interessierende Strukturen hervorgehoben und andere hintereinander liegende Objekte überblendet gezeichnet werden, obwohl diese für den Computer jederzeit differenziert aufschlüsselbar sind. Zur Visualisierung solcher hinter- einander liegender Objekte behelfen wir uns mit der planaren Darstellung in der entsprechenden axialen, sagittalen oder koronaren Projektion. Aus diesem Grunde wird intraoperativ für die navigiert kontrollierte Chirurgie die planare Projektion - teilweise kombiniert mit einem virtuellen $3 \mathrm{D}$-Modell - bevorzugt.

Mit Hilfe einer patientenkopfgetrackten Bildfusion verschiedener 3D-Daten- 


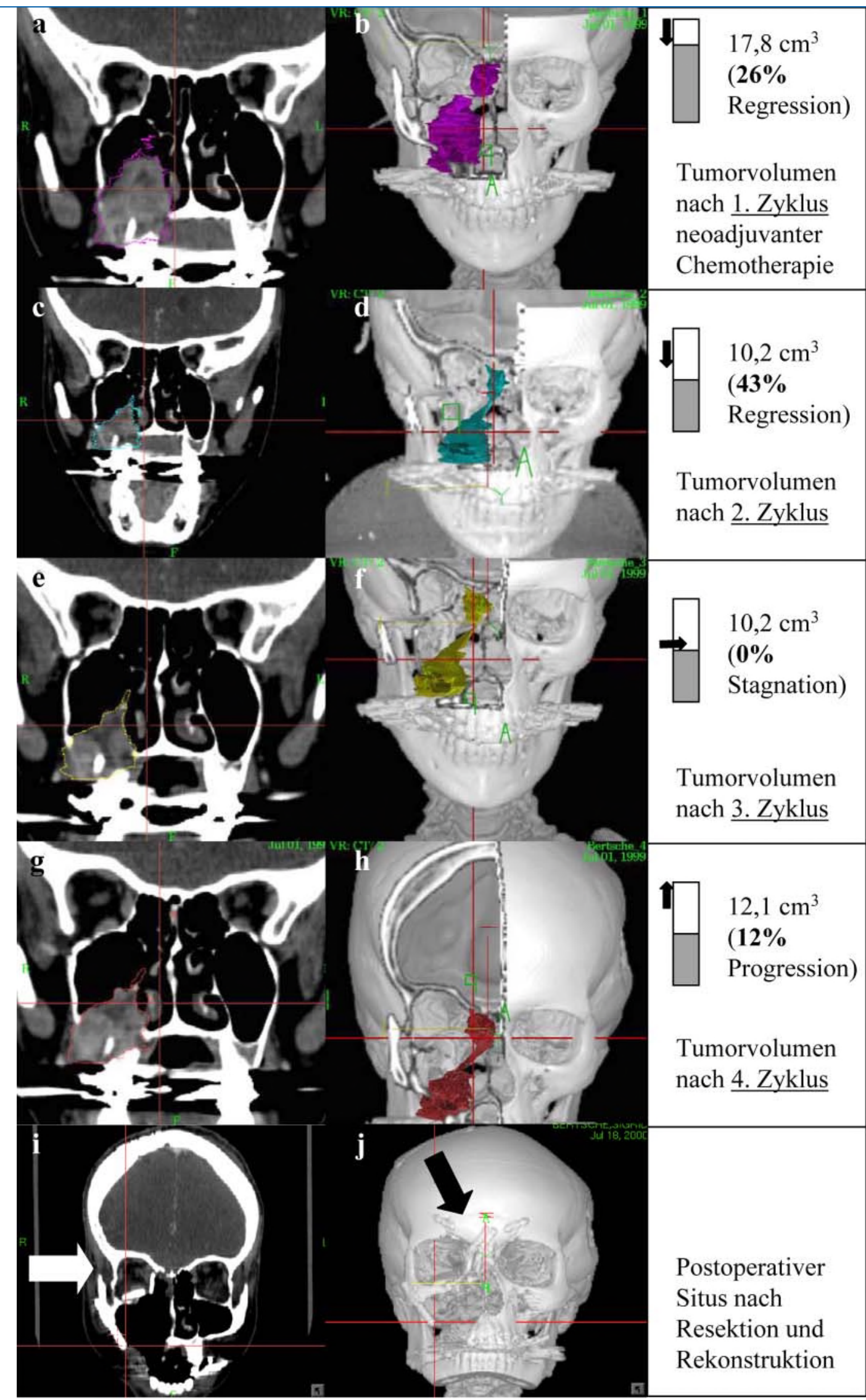

Abb. 3a-j $<$ Projektion eines virtuellen 3D-Modells von einem Nasennebenhöhlentumor (• Tab. 1, Fall 2) unter Berücksichtigung des interpolierten Tumorvolumens jeweils in einem CT-Datensatz: a, c, $\mathbf{e , g}$, i koronare Schichtung und $\mathbf{b}, \mathbf{d}, \mathbf{f}, \mathbf{h}, \mathbf{j}$ im 3D-Modell. Die prozentuale Bestimmung der Tumorregression bzw. -progression erfolgte ausgehend vom ursprünglichen Tumorvolumen zum Zeitpunkt der Erstdiagnose $(23,9 \mathrm{~cm} 3) \mathrm{im}$ Verhältnis zum jeweils aktuell errechneten Tumorvolumen anhand der virtuellen Modellanalyse. Der Pfeil in i zeigt auf die "splitbone grafts" zur navigierten Rekonstruktion der Orbita; der Pfeil in j zeigt auf den osteosynthetisch reimplantierten frontalen Knochendeckel nach subfrontalem Zugang nach Raveh 
sätze können anhand der virtuellen Modellanalyse bei Malignomen die Therapieergebnisse nichtchirurgischer Therapieverfahren (Chemotherapie, interstitielle und perkutane Strahlentherapie) sowie im Tumor-Follow-up als Monitoring objektiviert werden. Ebenso ist es möglich, die originären Tumorgrenzen und die chirurgische Rekonstruktion $[6,14]$ intraoperativ zu validieren. Die virtuelle Modellanalyse mit Hilfe der patientenkopfgetrackten Bildfusion ist damit das derzeit einzig sichere Verfahren für eine Objektivierung von Therapieergebnissen beim klinischen Monitoring oder auch intraoperativ.

Jedoch limitiert bislang immer noch der nicht zu vernachlässigende Zeitaufwand für den Segmentierungsprozess den Einsatz der virtuellen Modellanalyse zur Objektivierung von Therapieergebnissen im Schädelbasisbereich auf besondere Indikationen: Ein im Weichteilgewebe glatt abgrenzbarer Tumor wie etwa eine solitäre Lungenmetastase lässt sich heute immer noch in einer planaren Schichtbildgebung sicher überwachen im Gegensatz zu einem dysmorph infiltrierend wachsenden Plattenepithelkarzinom im Bereich des Splanchiokraniums. Gerade im Grenzbereich zwischen Knochen und Weichteilgewebe im Schädelbasisbereich zeigen infiltrierend wachsende Tumoren wie Plattenepithelkarzinome ein intramural uneinheitliches Wachstum, sodass eine umfassende räumliche Beurteilung sämtlicher Tumorgrenzflächen nur an einem Volumenmodell sinnvoll erscheint.

Sowohl für ein Downstaging als auch zur Planung der Tumorresektionsgrenzen und für die Defektrekonstruktion halten wir daher für solche lokalisierten Karzinome eine virtuelle Modellanalyse für sehr hilfreich und empfehlenswert. Diese kann, wie in unserem Beispiel gezeigt (- Tab. 1, Fall 6), auch bei der Berechnung eines optimalen osteoplastischen Knochendeckels von Vorteil sein, um ein unnötiges osteoklastisches Vorgehen zu vermeiden.

Die stetige Weiterentwicklung neuer graphischer Algorithmen, immer schnellere Rechnerleistungen und die immer höher werdende Punktgenauigkeit radiologischer Schichtbildverfahren werden in den nächsten 10 Jahren zu einer weiteren Verbreitung der Technik der virtuellen
Endoskopie in klinischer Diagnostik und Therapieplanung führen.

\section{Fazit für die Praxis}

\section{Anhand patientenkopfgetrackter virtu- eller Modelle lassen sich an unübersicht- lichen Regionen wie im Schädelbasisbe- reich Therapieergebnisse objektivieren und überwachen.}

\section{Korrespondierender Autor \\ Prof. Dr. J. Schipper \\ Universitätsklinik für Hals-, Nasen- und Ohrenheilkunde und Poliklinik Universitätsklinikum Freiburg Killianstraße 5, 79106 Freiburg schipper@hno1.ukl.uni-freiburg.de}

Interessenkonflikt. Es besteht kein Interessenkonflikt. Der korrespondierende Autor versichert, dass keine Verbindungen mit einer Firma, deren Produkt in dem Artikel genannt ist, oder einer Firma, die ein Konkurrenzprodukt vertreibt, bestehen. Die Präsentation des Themas ist unabhängig und die Darstellung der Inhalte produktneutral.

\section{Literatur}

1. Altobelli DE, Kikinis R, Mulliken JB, Cline $H$, Lorensen W, Jolesz F (1993) Computer-assisted three-dimensional planning in craniofacial surgery. Plast Reconstr Surg 92(4): 576-585

2. Cline HE, Lorensen WE, Kikinis R, Jolesz F (1990) Three-dimensional segmentation of MR images of the head using probability and connectivity. J Comput Assist Tomogr 14(6): 1037-1045

3. Cline HE, Lorensen WE, Ludke S, Crawford CR, Teeter BC (1988) Two algorithms for the three-dimensional reconstruction of tomograms. Med Phys 15(3): 320-327

4. Ecke U, Maurer J, Boor S, Khan M, Mann WJ (2003) Fehlerquellen der Navigation in der lateralen Schädelbasischirurgie. HNO 51: 386-393

5. Fried MP, Moharir VM, Shinmoto H, Alyassin AM, Lorensen WE, Hsu L, Kikinis R (1999) Virtual laryngoscopy. Ann Otol Rhinol Laryngol 108(3): 221226

6. Gellrich NC, Schramm A, Hammer B, Rojas S, Cufi D, Lagrèze W, Schmelzeisen R (2002) Computer-assisted secondary reconstruction of unilateral posttraumatic orbital deformity. Plast Reconstr Surg 110: 1417-1429

7. Grevers G, Assal J, Vogt T, Wilimzig C (1991) Threedimensional magnetic resonance imaging in skull base. Am J Otolaryngol 12: 139-145

8. Herman GT, Liu HK (1977) Display of three-dimensional reconstruction: a method of measuring temporal bone structures including the length of cochlea. Ann Otol Rhinol Laryngol 98: 155-160

9. Kaus MR, Warfield SK, Nabavi A, Black PM, Jolesz FA, Kikinis R (2003) Automated segmentation of MR images of brain tumors. Radiology 218(2): 586-591
10. Kober H, Nimsky C, Vieth J, Fahlbusch R, Ganslandt $\mathrm{O}$ (2002) Co-registration of function and anatomy in frameless stereotaxy by contour fitting. Stereotact Funct Neurosurg 79(3-4): 272-283

11. Majdani $O$, Leinung $M$, Lenarz $T$, Heermann $R$ (2003) Navigationsgestützte Chirurgie im Kopfund Hals-Bereich. Laryngorhinootologie 82: 632644

12. Miga MI, Sinha TK, Cash DM, Galloway RL, Weil RJ (2003) Cortical surface registration for image-guided neurosurgery using laser-range scanning. IEEE Trans Med Imaging 22(8): 973-985

13. Moharir VM, Fried MP, Vernick DM et al. (1998) Computer-assisted three-dimensional reconstruction of head and neck tumors. Laryngoscope 108: 1592-1598

14. Schipper J, Ridder GJ, Spetzger U, Teszler CB, Fradis M, Maier W (2004) Individual prefabricated titanium implants and titanium mesh in skull base reconstructive surgery. Eur Arch Otorhinolaryngol 261(5): 282-290

15. Schipper J, Maier W, Arapakis I, Spetzger U, Tatagiba M, Laszig R (2005) Informationsassistierte Chirurgie als ein transdisziplinäres Operationsverfahren. Laryngorhinootologie 84: 165-170

16. Schramm A, Gellrich NC, Naumann S, Bühner U, Schön R, Schmelzeisen R (1999) Non-invasive referencing in computer assisted surgery. Med Biol Eng Comp (Suppl): 644-645

17. Soler L, Delingette $H$, Malandain G et al. (2001) Fully automatic anatomical, pathological, and functional segmentation from CT scans for hepatic surgery. Comput Aided Surg 6(3): 131-142 